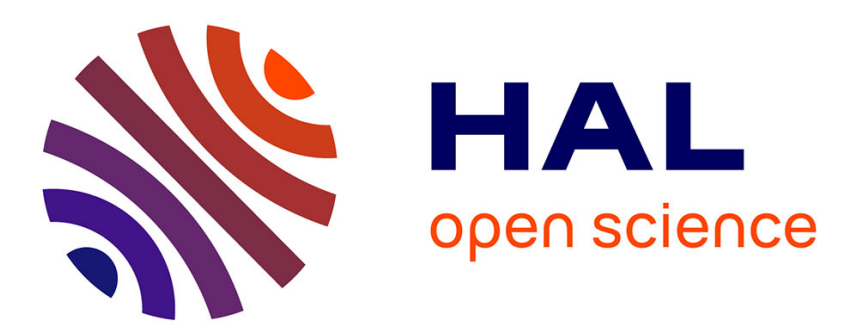

\title{
Regulation of kinase activity by diffusion and feedback
}

Bogdan Kazmierczak, Tomasz Lipniacki

\section{To cite this version:}

Bogdan Kazmierczak, Tomasz Lipniacki. Regulation of kinase activity by diffusion and feedback. Journal of Theoretical Biology, 2009, 259 (2), pp.291. 10.1016/j.jtbi.2009.03.016 . hal-00554592

\section{HAL Id: hal-00554592 \\ https://hal.science/hal-00554592}

Submitted on 11 Jan 2011

HAL is a multi-disciplinary open access archive for the deposit and dissemination of scientific research documents, whether they are published or not. The documents may come from teaching and research institutions in France or abroad, or from public or private research centers.
L'archive ouverte pluridisciplinaire HAL, est destinée au dépôt et à la diffusion de documents scientifiques de niveau recherche, publiés ou non, émanant des établissements d'enseignement et de recherche français ou étrangers, des laboratoires publics ou privés. 


\section{Author's Accepted Manuscript}

Regulation of kinase activity by diffusion and feedback

Bogdan Kazmierczak, Tomasz Lipniacki

PII:

S0022-5193(09)00113-1

DOI: doi:10.1016/j.jtbi.2009.03.016

Reference:

YJTBI 5489

To appear in: $\quad$ Journal of Theoretical Biology

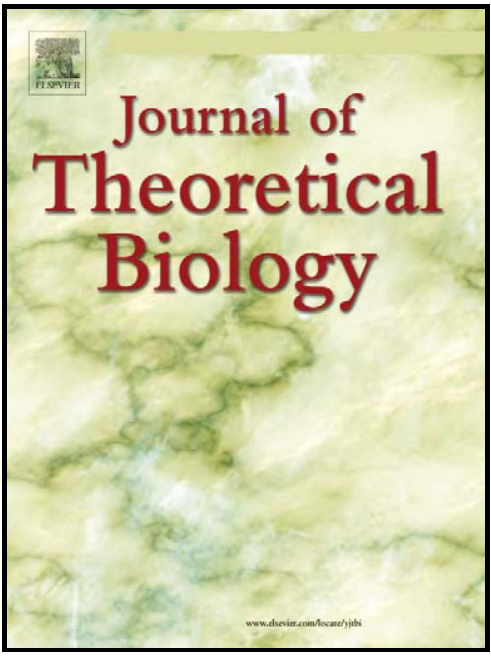

www.elsevier.com/locate/yjtbi

Received date: $\quad 11$ November 2008

Revised date: $\quad 18$ February 2009

Accepted date: $\quad 4$ March 2009

Cite this article as: Bogdan Kazmierczak and Tomasz Lipniacki, Regulation of kinase activity by diffusion and feedback, Journal of Theoretical Biology (2009), doi:10.1016/j.jtbi.2009.03.016

This is a PDF file of an unedited manuscript that has been accepted for publication. As a service to our customers we are providing this early version of the manuscript. The manuscript will undergo copyediting, typesetting, and review of the resulting galley proof before it is published in its final citable form. Please note that during the production process errors may be discovered which could affect the content, and all legal disclaimers that apply to the journal pertain. 


\title{
Regulation of kinase activity by diffusion and feedback
}

\author{
Bogdan Kazmierczak, Tomasz Lipniacki
}

February 18, 2009

Institute of Fundamental Technological Research, Warsaw, Poland.

Running title: Regulation of kinase activity by diffusion and feedback

\section{Corresponding author:}

Tomasz Lipniacki

Institute of Fundamental Technological Research,

Polish Academy of Sciences,

Swietokrzyska 21, 00-049 Warsaw, Poland

E-mail: tomek@rice.edu and tlipnia@ippt.gov.pl 


\section{Abstract}

In living cells proteins motilities regulate the spatiotemporal dynamics of molecular pathways. We consider here a reaction-diffusion model of mutual kinase-receptor activation showing that the strength of positive feedback is controlled by the kinase diffusion coefficient. For high diffusion, the activated kinase molecules quickly leave the vicinity of the cell membrane and cannot efficiently activate the receptors. As a result, in a broad range of parameters, the cell can be activated only if the kinase diffusion coefficient is sufficiently small. Our simple model shows that change in the motility of substrates may dramatically influence the cell responses.

Keywords: reaction-diffusion system, signal transduction, positive feedback, kinase activation, membrane receptors, protein motilitity.

\section{Introduction}

Regulatory networks process cellular signals in time and space enabling cell self-organization (see Kholodenko 2006 and Karsenti 2008 for reviews). The temporal dynamics is coupled with spatial gradients of concentrations or activity. For example, kinase cascades can emerge from receptors and transmit signals from the cell membrane to the nucleus. In this case the gradient of active kinase activity develops since phosphorylation and dephosphorylation proceed at different cellular locations, respectively cell membrane and cell volume. Due to the estimations of Brown and Kholodenko (1999), basing on measured values of protein diffusion coefficients and phosphatase activities, gradients of kinase activity are potentially very large. In a simple system in which kinase molecules are phosphorylated at the cell membrane and dephosphorylated by a phosphatase molecules located homogeneously in the cell cytosol (analyzed by Brown and Kholodenko 1999) small diffusion implies high gradient and low kinase activity in the cell center. The problem of receptor-kinase interaction has been also studied in the context of diffusion with obstacles in the stochastic numerical simulations of bacterial chemotaxis (Lipkow et al. 2005). One of the conclusions of Lipkow et al. 2005 is that crowding results in a fall of the apparent diffusion coefficient and at the anterior end, where CheY is phosphorylated, the local concentration of CheYp increases and therefore accelerates the response of the anterior close motor. At the other, posterior, end of the cell, the local CheYp concentration is reduced by the need to diffuse through the obstacles and the responses of motors in this region is consequently delayed. 
Here we consider a similar model to the one analyzed by Brown and Kholodenko (1999), but assume the mutual receptor - kinase activation. Membrane receptors can bind extracellular ligands, that leads to cascade of molecular processes inside the cell and formation of the active receptor complex. In many cases, receptor activation requires phosphorylation. Almost all G-protein coupled receptors (GPCRs) are regulated by phosphorylation, see Tobin (2008) for review. Engagement of immunoreceptors (TCR, BCR, FcR) leads to activation of different members of the Src kinase family, which includes Lck (for T-cell, Housden et al. 2003), Fyn and Lyn (for B and mast cells, Gauld and Cambier 2004). Src kinases then phosphorylate immunoreceptor tyrosine-based activation motifs (ITAMs) contained within the immunoreceptors themselves or in receptor-associated molecules, see Abram and Lowell (2007) for review. This may lead to positive feedback, in which active receptors send signal to kinase and in turn are activated by the same kinase species or by one of the downstream kinases. In this study we consider the simplest situation, in which receptors are activated by the same kinase species they activate.

We will show, that in the case of mutual receptor - kinase activation, in a broad range of parameters controlling the process, the cell becomes activated only if the kinase diffusion is sufficiently small. For large diffusion, the activated kinase molecules quickly leave vicinity of cell membrane, and the positive feedback coupling kinases with receptors becomes inefficient.

\section{Model formulation}

As said, we will assume that membrane receptors bind extracellular ligands, that leads to a cascade of processes and receptor activation. At constant extracellular cytokine concentration, a steady state uniform surface concentration of ligand-bound receptors $P=$ const is established. We will consider two cases. Firstly, following Brown and Kholodenko (1999) we will assume that all ligandbound receptors are active. Secondly, we will assume that the limiting step in the formation of the active receptor complex is its phosphorylation by the kinase. In turn, active receptors may activate kinase molecules, that defines the positive feedback in the regulation process. The activated kinase may freely diffuse over entire cell volume, where they are inactivated by uniformly distributed phosphatases.

The cell will be modeled geometrically as a ball $B\left(0, r_{0}\right)$ of radius $r_{0}$, centered at the origin of the coordinate system. We restrict to the spherically symmetric case and we will use the following notation : 
$K(t, r)$ - the concentration of the active kinase

$Q=$ const - the total concentration of the kinase

$R(t)$ - the surface concentration of the active receptors

$P=$ const - the total surface concentration of the ligand bound receptors (active and inactive)

$\Phi_{K}(t)$ - the flux of the active kinase

The active kinase concentration satisfies

$$
\frac{\partial K}{\partial t}=d_{1} \nabla^{2} K-b_{1} K
$$

where $b_{1}>0$ is the kinase dephosphorylation rate due to the action of uniformly distributed phosphatases. The flux of the active kinase results from its phosphorylation by the surface receptors implying the Robin type boundary condition,

$$
\Phi_{K}=a_{1} R\left(Q-K_{b}\right)=d_{1} \mathbf{n} \cdot(\nabla K)_{b}
$$

where $\mathbf{n}$ is a unit vector normal to cell surface and subscript $b$ denotes the boundary value for $\left(r=r_{0}\right)$.

As already mentioned, regarding the receptors activation we will consider two different cases;

(1) Activity of the receptors is independent of intracellular processes, but is controlled by binding and dissociation of some extracellular ligand (present at the constant concentration). In such a case we may assume that all the bound receptors are persistently active i.e. $R(t)=$ const $=P$.

(2) Alternatively, we may assume that the limiting step in the receptor activation is its phosphorylation by the kinase, that defines the positive feedback in receptor-kinase activation,

$$
\frac{d R}{d t}=a_{2} K_{b}(P-R)-b_{2} R
$$

In the further consideration we will assume that all the reaction rate coefficients, $a_{1}, a_{2}, b_{1}, b_{2}$, and diffusion constant $c_{1}$ are positive. In the non-dimensional units $\tau=t b_{1}, \rho=r / r_{0}$, Eq. 1 reads

$$
\frac{\partial K}{\partial \tau}=d \nabla^{2} K^{*}-K^{*}
$$

where $d=d_{1} /\left(b_{1} r_{0}^{2}\right)$ plays the role of the non-dimensional diffusion coefficient and $K^{*}=K / Q$. We may thus rewrite Eqs. 2 and 3 as

$$
a R^{*}\left(1-K_{b}^{*}\right)=d \mathbf{n} \cdot\left(\nabla K^{*}\right)_{b},
$$




$$
\frac{d R^{*}}{d \tau}=q K_{b}^{*}\left(P^{*}-R^{*}\right)-b R^{*}
$$

where $a=a_{1} P / b_{1}, R^{*}=R /\left(r_{0} Q\right), q=a_{2} Q / b_{1}, b=b_{2} / b_{1}$ and $P^{*}=P /\left(r_{0} Q\right)$. From now on, for the sake of simplicity all the asterisks will be omitted. Let us notice that $\rho \in[0,1], K \in[0,1]$, $R \in[0, P]$ and $a, b, d, q$ and $P$ are real and nonnegative.

\section{Results}

\subsection{Limit of infinite diffusion $d \rightarrow \infty, K=K(\tau), R=R(\tau)$.}

For the infinite diffusion, the active kinase concentration is uniform, and the system of Eqs. 4-6 is equivalent to the system of two ordinary equation, for $K(\tau), R(\tau)$,

$$
\begin{gathered}
\frac{d K}{d \tau}=3 a R(\tau)(1-K(\tau))-K(\tau) \\
\frac{d R}{d \tau}=q K(P-R(\tau))-b R(\tau) .
\end{gathered}
$$

Formally, we can obtain the above system by integrating Eq. 4 over the ball $B(0,1)$ and using the Gauss theorem. Let us note that the compact region $D:=[0,1] \times[0, P]$ is invariant with respect to the flow generated by the above system. That is to say, if $\{K(0), R(0)\} \in D$, then for arbitrary $\tau \geq 0,\{K(\tau), R(\tau)\} \in D$. System $7-8$ has two steady states: $\left\{K_{1}, R_{1}\right\}=\{0,0\}$ and $\left\{K_{2}, R_{2}\right\}$, where

$$
K_{2}=\frac{3 q a P-b}{q(3 a P+1)}, \quad R_{2}=\frac{3 q a P-b}{3 a(b+q)}
$$

For $b<3 q a P$ the steady state point $\left\{K_{2}, R_{2}\right\}$ is stable, while the point $\left\{K_{1}, R_{1}\right\}$ is unstable, for $b \geq 3 q a P, K_{2}<0, R_{2}<0$ and the point $\left\{K_{2}, R_{2}\right\}$ is unstable, while the $\left\{K_{1}, R_{1}\right\}$ is stable. In other words, restricting to subdomain $D$, the system has one stable steady state $\{0,0\}$, for $b \geq 3 q a P$, or $\left\{K_{2}, R_{2}\right\}$ for $b<3 q a P$.

In the case without the feedback, (i.e. when $R(\tau)=P$ ) Eq. 7 can be solved analytically, 


$$
K(\tau)=\left(K(0)-\frac{3 a P}{3 a P+1}\right) \exp [(1+3 a P) \tau]+\frac{3 a P}{3 a P+1}
$$

and has unique stable state $K_{3}=3 a P /(1+3 a P)$.

\subsection{Finite diffusion - steady state analysis}

\section{(1) Case without feedback}

In spherical coordinates Eq. 4 reads

$$
\frac{\partial K}{\partial \tau}=d \frac{1}{\varrho^{2}} \frac{\partial}{\partial \varrho}\left(\varrho^{2} \frac{\partial K}{\partial \varrho}\right)
$$

and has unique steady state solution

$$
K(\varrho)=\frac{K_{c}\left(e^{\alpha \varrho}-e^{-\alpha \varrho}\right)}{2 \varrho \alpha},
$$

where $\alpha=d^{-1 / 2}$ and $K_{c}=K(0)$. Using Eq. 5 (with $R=P$ ) and Eq. 12 we may calculate boundary value $K_{b}=K(1)$

$$
K_{b}=\frac{a P \alpha^{2}\left(e^{2 \alpha}-1\right)}{\left.1+\alpha-a \alpha^{2} P+e^{2 \alpha}\left(\alpha+a \alpha^{2} P-1\right)\right)},
$$

and then

$$
K_{c}=2 K_{b} \alpha /\left(e^{\alpha}-e^{-\alpha}\right)=\frac{2 a P \alpha^{3} e^{\alpha}}{\left.1+\alpha-a \alpha^{2} P+e^{2 \alpha}\left(\alpha+a \alpha^{2} P-1\right)\right)} .
$$

In the limit of infinite diffusion $\alpha \rightarrow 0$, the active kinase distribution is uniform, $K(\varrho) \equiv K_{c}$, with $K_{c}=K_{3}$. The total amount of active kinase $K_{t o t}$ is

$$
K_{t o t}=4 \pi \int_{0}^{1} \varrho^{2} K(\varrho) d \varrho=4 \pi K_{c} \frac{\alpha \cosh (\alpha)-\sinh (\alpha)}{\alpha^{3}} .
$$

Differentiating $K_{b}, K_{c}$ and $K_{\text {tot }}$ several times with respect to $\alpha$, one can show that for $a>0$ and $P>0$ :

1) $K_{b}$ is a monotonically increasing function of $\alpha=d^{-1 / 2}$,

2) $K_{c}$ and $K_{t o t}$ are monotonically decreasing functions of $\alpha$. 
Large diffusion enhances the flux of the active kinase from the cell membrane towards the cell center. Thus, as one could expect, both $K_{c}$ and $K_{t o t}$ are growing functions of diffusion coefficient. Simultaneously, the active kinase concentration close to the boundary decreases with increasing diffusion, Fig. 1.

\section{(2) Case with feedback}

In the case with feedback the spatial profile of $K(\varrho)$ is the same as in the case without feedback; Eqs. 5-6 gives us two values of $K_{b}$ (or $K_{c}=K_{b} * 2 \alpha /\left(e^{\alpha}-e^{-\alpha}\right)$ ) corresponding to one stable and one unstable steady state solution

$$
K_{c 1}=0, \quad K_{c 2}=\frac{2 \alpha e^{\alpha}\left(b e^{2 \alpha}(1-\alpha)-b(\alpha+1)+q a P \alpha^{2}\left(e^{2 \alpha}-1\right)\right)}{q\left(e^{2 \alpha}-1\right)\left(1+\alpha-a \alpha^{2} P+e^{2 \alpha}\left(\alpha+a \alpha^{2} P-1\right)\right)} .
$$

The stable solution $K(\varrho)$ is given by

$$
K(\varrho)=\frac{K_{c}\left(e^{\alpha \varrho}-e^{-\alpha \varrho}\right)}{2 \varrho \alpha} .
$$

where $K_{c}=\max \left(K_{c 1}, K_{c 2}\right)$, i.e. for $K_{c 2}>0$ the stable solution is positive, while for $K_{c 2}<0$, $K(\varrho) \equiv 0$. The global stability (with respect to a perturbation without spherical symmetry) of $K(\varrho)$ solution is proved in the Appendix.

Let us note, that in the limit of the infinite diffusion coefficient $d \rightarrow \infty$, i.e. $\alpha \rightarrow 0, K(\varrho) \equiv$ $K_{c}=K_{2}$ and thus one obtains the same solution as given in Eq. 9. In the opposite limit $d \rightarrow 0$ (i.e. $\alpha \rightarrow \infty), K_{b}=1, K_{c}=0, K_{t o t}=0$. In further analysis we set $q=a=P=1$, and consider the steady state kinase activity profiles with respect to two nondimensional parameters: $\alpha=d^{-1 / 2}$, and $b$ (receptor inactivation constant).

In Fig. 2 we analyze the dependence of the stable steady state $K(\varrho)$ on the diffusion parameter $\alpha=d^{-1 / 2}$. With respect to the receptor dephosphorylation coefficient $b$, we may distinguish three cases, shown in Panels A, B and C. For small $b$ (Panel A), the dependence of active kinase concentration profiles $K(\varrho)$ on $\alpha$ is similar as in the case without feedback; the larger is $\alpha$, the steeper is the active kinase profile, with higher value at the boundary and the lower value in the cell center. Qualitatively different is the case shown in Panel B for larger values of $b$. For $b=2.5$, the active kinase concentration is higher across the whole cell for some finite diffusion $(\alpha=2)$ than for the infinite diffusion $(\alpha=0)$. In the case of large dephosphorylation parameter (Panel $\mathrm{C}, b=4)$ for infinite, or large diffusion, $K(\varrho) \equiv 0$, while for smaller diffusion $K(\varrho)>0$. This somehow surprising effect is due to the fact that the strength of positive feedback is controlled by the diffusion. For 
small diffusion activated kinase remain longer in the vicinity of the membrane and may activate the receptors more effectively.

In Figs. $3 \mathrm{~A}$ and $3 \mathrm{~B}$ we analyze $K_{b}(\alpha)$ and $K_{c}(\alpha)$ for four different values of $b$. As can be expected,$K_{b}$ is a growing function of $\alpha$. For small $b, K_{c}$ decreases with growing $\alpha$ (as in the case without feedback), however for larger $b, K_{c}(\alpha)$ has a maximum for some $\alpha_{m}(b)>0$. The existence of such an "optimal" $\alpha_{m}$ is due to interplay of two counter-effects:

(1) large diffusion ( $\operatorname{small} \alpha$ ) speeds translocation of active kinase, so they have a larger chance to remain phosphorylated until they reach the cell center,

(2) simultaneously large diffusion attenuates the positive feedback coupling kinases with receptors.

In contrast to the case without feedback, the total amount of active kinase $K_{\text {tot }}(\alpha)$ for large $b$ has maximum for some $\alpha_{m}^{\prime}(b)>\alpha_{m}(b)>0$. Let us note also, that both $\alpha_{m}^{\prime}(b)$ and $\alpha_{m}(b)$ are growing functions of $b$, diverging logarithmically to infinity with $b$.

As shown in Fig. 4 there are unbounded parameter domains $D_{c}$ and $D_{t o t}$ in $(\alpha, b)$ plane for which respectively $\Delta_{c}:=K_{c}(\alpha, b)-K_{c}(0, b)>0$ and $\Delta_{t o t}:=K_{t o t}(\alpha, b)-K_{t o t}(0, b)>0$. Since for finite diffusion $K_{b}(\alpha)>K_{c}(\alpha): D_{c} \subset D_{\text {tot }}$. In the case of the infinite diffusion $(\alpha=0)$, the positive solutions $K(\rho, \alpha, b)>0$ are restricted to domain $b<3$. In the case of finite diffusion, for arbitrarily large $b$ there exists such $\alpha(b)$ that $K(\rho, \alpha, b)>0$.

\section{Discussion}

Dynamics of molecular pathways is determined by both, chemical reaction rules and localization of substrates that in turn is governed by diffusion or transport. We considered here a simple theoretical model of mutual receptor-kinase activation in which the kinase molecules, are phosphorylated by the receptors at the cell membrane and may freely diffuse in the cell volume, where they are dephosphorylated with time- and space-independent dephosphorylation rate. The positive feedback, considered in the model, arises since activated kinase may in turn activate receptors.

In the case without feedback, active kinase concentration in the cell center and the total amount of active kinase are monotonically growing functions of diffusion coefficient. However, the presence of the positive feedback causes that the concentration of the active kinase in the cell volume is a nontrivial function of the diffusion coefficient. The active kinase concentration in the cell center depends on the two opposite, diffusion controlled effects: the kinase activity profile along the cell 
radius is flatter for large diffusion, and the strength of positive feedback controlling active kinase concentration at the boundary is stronger for small diffusion. As a result in a broad range of parameters $a, q, P$ and $b$ controlling mutual kinase-receptors activation and inactivation rates the maximum value of the active kinase concentration in the cell center is reached for some finite value of the kinase diffusion coefficient. Moreover, for the large receptor inactivation rate $(b \geq 3 q a P)$ the active kinase concentration is everywhere zero for the infinite diffusion, but it is positive for the sufficiently small diffusion. Interestingly, for constant $a, q$ and $P$, even for arbitrarily large inactivation coefficient $b$, there exists a positive steady state solution for the active kinase concentration if the diffusion is sufficiently small.

In living cells the diffusion and thus spatiotemporal localization of substrates can be controlled in a number of ways; Molecules can bind to a larger molecules of lower motility called buffers, or to the cell membrane and other structural elements directly or with help of the, so called, anchoring proteins. On the cell membrane receptors can form larger complexes of lower motility, or get localized within lipid rafts. Relevant to our model, cell membrane can create microdomains which trap signalling molecules, like Lck kinase, that activates TCR receptors, Douglass and Vale (2005). Major simplification of our study is that it does not account for macromolecular crowding and presence of diffusion obstacles within the cell (organelle, cellular structures). The macromolecular crowding may have non-trivial effect on molecular association in the cell, possibly increasing its rate by limiting the volume in which molecules are free to diffuse (see Minton 2001 and Zimmerman and Minton 1993).

Our simple model provides an example in which diffusion controls the strength of the feedback regulation and thus the dynamics of kinase activation. In the considered model, for a broad range of parameters the cell can be activated only when the kinase diffusion coefficient is sufficiently small, i.e. when reacting kinases and receptors are well collocalized that enables their mutual activation.

\section{$5 \quad$ Acknowledgments}

This study was supported by Polish Committee for Scientific Research Grants No. 4 T07A 00130 and No. 1P03A01230. 


\section{References}

Abram, C.L., Lowell, C.A., 2007. Convergence of immunoreceptor and integrin signaling. Immunological Reviews 218:29-44.

Brown, G.C., Kholodenko, B.N., 1999. Spatial gradients of cellular phospho-proteins, FEBS Lett. 457:452-454.

Douglass, A.D., Vale, R. D., 2005. Single-molecule microscopy reveals plasma membrane microdomains created by protein-protein networks that exclude or trap signaling molecules in T cells, Cell 121:937-950.

Gauld, S.B., Cambier, J.C., 2004. Src-family kinases in B-cell development and signaling. Oncogene 23:8001-8006.

Housden, H.R., Skipp, P.J.S., M. P. Crump, M.P., Broadbridge, R.J., Crabbe, T., Perry, M.J., Gore, M.G., 2003. Investigation of the kinetics and order of tyrosine phosphorylation in the T-cell receptor $\xi$ chain by the protein tyrosine kinase Lck, Eur. J. Biochem. 270, 2369-2376.

Karsenti E., 2008. Self-organization in cell biology: a brief history. Nat. Rev. Mol. Cell Biol. 9:255-262.

Kholodenko B.N., 2006. Cell-signalling dynamics in time and space. Nat. Rev. Mol. Cell Biol. $7: 165-174$.

Lipkow, K., Andrews, S.S., Bray, D., 2005. Simulated diffusion of phosphorylated CheY through the cytoplasm of Escherichia coli. J. Bacteriol 187:45-53.

Minton, A.P., 2001. The influence of macromolecular crowding and macromolecular confinement on biochemical reactions in physiological media. J. Biol. Chem. 276:10577-10580.

Pao C.V., 1992. Nonlinear Parabolic and Elliptic Equations. Plenum Press, New York and London.

Tobin, A.B., 2008. G-protein-coupled receptor phosphorylation: where, when and by whom. British Journal of Pharmacology 153: S167-S176.

Zimmerman, S.B., Minton, A.P., 1993. Macromolecular crowding: Biochemical, biophysical, and physiological consequences, Annu. Rev. Biophys. Biomol. Struct. 22, 27-65.

\section{Figure captions}

Fig. 1. The case without feedback. Profiles of active kinase concentration $K(\rho)$ for different values of $\alpha=d^{-1 / 2}$. For all plots the remaining parameters are fixed: $a=P=1$. 
Fig. 2. The case with feedback. Profiles of active kinase concentration $K(\rho)$ for different values of $\alpha=d^{-1 / 2}$. Three qualitatively different cases corresponding to three different value of nondimensional receptor dephosphorylation rate $b$ are considered; Panel $\mathrm{A},(b=1, \alpha=0,2,4)$, Panel B, $(b=2.5, \alpha=0,2,4)$, and Panel $\mathrm{C}(b=4, \alpha=0,3.5,6)$. For all plots the remaining parameters are fixed: $q=a=P=1$.

Fig. 3. The case with feedback. Concentration of active kinase at the boundary $K_{b}$ (Panel A), in the cell center $K_{c}$ (Panel B), and total amount of active kinase $K_{\text {tot }}$ (Panel C) as a function of $\alpha=d^{-1 / 2}$. For all plots the remaining parameters are fixed: $q=a=P=1$.

Fig. 4. The case with feedback. Dependence of active kinase concentration in the cell center (Panel A) and total amount of active kinase (Panel B) with respect to $\alpha=d^{-1 / 2}$ and nondimensional receptor dephosphorylation rate $b$. In Panel $\mathrm{A}$ we may distinguish three domains in $(\alpha, b)$ plane in which respectively, $K_{c}(\alpha, b)=0 ; \Delta_{c}=K_{c}(\alpha, b)-K_{c}(0, b)>0 ; \Delta_{c} \leq 0$. In domain $\Delta_{c}>0$, isolines of $\Delta_{c}$ are shown. Similarly, in Panel B we may distinguish three domains in which respectively, $K_{t o t}(\alpha, b)=0 ; \Delta_{t o t}=K_{t o t}(\alpha, b)-K_{t o t}(0, b)>0 ; \Delta_{t o t} \leq 0$. In domain $\Delta_{t o t}>0$, isolines of $\Delta_{t o t}$ are shown. For both plots the remaining parameters are fixed: $q=a=P=1$.

\section{Appendix. Stability of spherically symmetric solutions}

Let $\widetilde{K}(\rho)$ and $\widetilde{R}=$ const denote the spherically symmetric stationary solution of system 4-6. For the initial data not coinciding with $(\widetilde{K}(\rho), \widetilde{R})$, the solution $(K(x, \tau), R(\tau))$ to system 4-6 will be in general different, i.e.

$$
K(x, \tau)=\tilde{K}(\rho)+\delta K(x, \tau), \quad R(x, \tau)=\widetilde{R}+\delta R(x, \tau) .
$$

Our task here is to examine the asymptotic in time behavior of the functions $K$ and $R$. We will confine here to initial data preserving the positivity of the functions $K$ and $R$. The equations for $\delta R$ and $\delta K$ read:

$$
\frac{\partial \delta K}{\partial \tau}=d \nabla^{2} \delta K-\delta K
$$

inside the sphere and

$$
\begin{aligned}
& \frac{d \delta R}{d \tau}=q \delta K_{b}(P-\widetilde{R})-\left(q \widetilde{K}_{b}+b\right) \delta R-q \delta K_{b} \delta R \\
& a \delta R\left(1-\widetilde{K}_{b}\right)-a \widetilde{R} \delta K_{b}-a \delta R \delta K_{b}=c \mathbf{n} \cdot \nabla \delta K_{b}
\end{aligned}
$$


on the sphere. Here we used the fact that $\widetilde{R}$ and $\widetilde{K}$ satisfy system $4-6$. For $\varepsilon>0$ an arbitrarily small positive number, let

$$
\delta k=\delta K \exp \varepsilon \tau, \quad \delta r=\delta R \exp \varepsilon \tau
$$

Then, the above equations can be written as:

$$
\begin{gathered}
\frac{\partial \delta k}{\partial \tau}=d \nabla^{2} \delta k-(1-\varepsilon) \delta k \\
\frac{d \delta r}{d \tau}=q(P-\widetilde{R}) \delta k_{b}-\left(q \widetilde{K}_{b}+b+q \delta k_{b} \exp (-\varepsilon \tau)-\varepsilon\right) \delta r \\
a \delta r(x, \tau)\left(1-\widetilde{K}_{b}\right)=d \mathbf{n} \cdot \nabla \delta k+a(\widetilde{R}+\delta r(x, \tau) \exp (-\varepsilon \tau)) \delta k_{b} .
\end{gathered}
$$

The idea of the stability proof is to construct a time independent sub- and supersolution pairs: $\left(-\delta r_{-}, \delta k^{-}\right)$and $\left(\delta r_{+}, \delta k^{+}\right)$. As $\varepsilon>0$ this will prove that $\delta R(x, \tau)$ and $\delta K(x, \tau)$ tend to 0 as $\tau \rightarrow \infty$.

Let

$$
\delta K^{ \pm}(\rho)= \pm \delta K_{ \pm b} \phi(\rho ; \alpha \varepsilon)
$$

where $\alpha \varepsilon=\left(\frac{1-\varepsilon}{d}\right)^{1 / 2}$ and

$$
\phi(\rho ; \alpha)=\frac{\left(e^{\alpha \varrho}-e^{-\alpha \varrho}\right)}{\varrho\left(e^{\alpha}-e^{-\alpha}\right)} .
$$

Let us recall that $\phi$ is an increasing function of $\rho$ and $\phi(1 ; \alpha)=1$ for all $\alpha \in(0, \infty)$. In fact

$$
\widetilde{K}(\rho)=\widetilde{K}_{b} \phi\left(\rho ; \alpha_{0}\right)
$$

Let

$$
\delta K_{-b}=\widetilde{K}_{b}-\psi(\varepsilon)
$$

where $\psi(\varepsilon) \searrow 0$ as $\varepsilon \rightarrow 0$. This function will be specified later. It follows from 23,24 and the continuity of the function $\phi(\rho ; \alpha \varepsilon)$ with respect to the parameter $\varepsilon$ that if $K(x, 0)>\eta>0$ for $|x| \leq 1$, we can find $\varepsilon>0$ so small that

$$
K(x, 0)>\widetilde{K}(\rho(x))-\delta K_{-b} \phi(\rho(x) ; \alpha \varepsilon)>0
$$

for all $x$ inside the sphere. Let $\delta K^{+}$be at least so large that $K(x, 0)<\widetilde{K}(\rho(x))+\delta K_{+b}(\rho(x))$, implying that $\delta K(x, 0)<\delta K_{+b}(\rho(x))$. Let 


$$
W_{R}=\frac{q(P-\widetilde{R})}{q \widetilde{K}_{b}-q \delta K_{-b}+b-\varepsilon}=\frac{q(P-\widetilde{R})}{q \psi(\varepsilon)+b-\varepsilon} .
$$

We will assume that

$$
\delta R_{ \pm}=\delta K_{ \pm b}\left(W_{R}+\varepsilon\right)
$$

Let us note that as

$$
(P-\widetilde{R}) / \widetilde{R}=b /\left(q \widetilde{K}_{b}\right),
$$

it follows from Eq.27 that:

$$
\delta R_{-}=\widetilde{R}-C_{2} \psi(\varepsilon)+\widetilde{C}_{2} \varepsilon+o(\psi(\varepsilon))+o(\varepsilon) .
$$

for some positive constants $C_{2}$ and $\widetilde{C}_{2}$. If, for small $\varepsilon \geq 0, \psi(\varepsilon) / \varepsilon \geq C_{3}>0$ with $C_{3}$ sufficiently large, then $\delta R_{-}<\widetilde{R}$. On the other hand, for any $R(0)>0$ we can find $\varepsilon>0$ sufficiently small such that $R(0)>\widetilde{R}-\delta R_{-}$. Obviously, we may also choose $\delta K_{+}$so large that $R(0)<\widetilde{R}+\delta R_{+}$.

Let

$$
\delta r_{ \pm}=\delta R_{ \pm}, \quad \delta k_{ \pm b}=\delta K_{ \pm b}
$$

Let $\delta k^{ \pm}(\cdot)$ be constant in time spherically symmetric solutions to Eq.19:

$$
\delta k^{ \pm}(\rho)= \pm \delta k_{ \pm b} \phi(\rho ; \alpha \varepsilon)
$$

We will prove that $\left(-\delta r_{-}, \delta k^{-}\right)$and $\left(\delta r_{+}, \delta k^{+}\right)$defined in 30 and 31 are respectively the sub- and supersolution pairs for system 19-21. So, after putting $\delta r=-\delta r_{-}$in Eq.20 we infer that the right hand side is positive if $\delta k_{b} \geq-\delta k_{-b}$. In the similar way, after putting $\delta r=\delta r_{+}$in Eq.20 we infer that the right hand side is negative if $\delta k_{b} \leq \delta k_{+b}$. It follows that if $\delta k_{+} \geq \delta k(x, \tau) \geq-\delta k_{-}$at the boundary, then $\delta r(x, \tau) \in\left(-\delta r_{-}, \delta r_{+}\right)$. We have thus to prove the corresponding properties of the functions $\delta k^{-}$and $\delta k^{+}$with respect to Eqs.19-21. First, as we said, the functions $\delta k^{ \pm}(\rho)$ satisfy Eq.19. So, to prove that $\delta k^{-}$, defined in $(22)$ is a subsolution it suffices to show, as $\left(\delta k^{-}\right)_{\mid B}^{\prime}=$ $w\left(\frac{1-\varepsilon}{d}\right)\left(-\delta k_{-b}\right)$, that for all $\delta r(x, \tau) \in\left(-\delta r_{-}, \delta r_{+}\right)$we have

$$
\delta r(x, \tau)\left(1-\widetilde{K}_{b}+\delta k_{-b}\right) \geq-\widetilde{R} \delta k_{-b}-a^{-1} w\left(\frac{1-\varepsilon}{d}\right) \delta k_{-b} .
$$

(See, chapter 2 of Pao 1992. Note that the coefficient by $\delta k_{b}$ in 21 is positive.) Here 


$$
w(s)=\phi_{, \rho}(\rho=1, s)
$$

is smooth and monotonically increasing function of $s$ for $s \geq(2 d)^{-1}$. As $\delta k_{-b}=\delta K_{-b}<\widetilde{K}_{b}$ then, putting $\delta r(x, \tau)=-\delta r_{-}$, we conclude that inequality 32 is implied by the inequality:

$$
\left(1-\widetilde{K}_{b}+\delta K_{-b}\right)\left[\frac{q(P-\widetilde{R})}{q \widetilde{K}_{b}-q \delta K_{-b}+b-\varepsilon}+\varepsilon\right]<\widetilde{R}+a^{-1} w\left(\frac{1-\varepsilon}{d}\right)
$$

where we used 27 . Now, due to $28,(P-\widetilde{R}) / \widetilde{R}=b /\left(q \widetilde{K}_{b}\right)$. Hence, we obtain the condition:

$$
b\left(1-\widetilde{K}_{b}+\delta K_{-b}\right) /\left(q \widetilde{K}_{b}-q \delta K_{-b}+b-\varepsilon\right)+O_{1}(\varepsilon)<\widetilde{K}_{b}+\frac{\widetilde{K}_{b}}{\widetilde{R}} a^{-1} w\left(\frac{1-\varepsilon}{d}\right) .
$$

As the spherically symmetric solution satisfies the equality

$$
a \widetilde{R}\left(1-\widetilde{K}_{b}\right)=w\left(\frac{1}{d}\right) \widetilde{K}_{b}
$$

then

$$
\begin{array}{r}
b\left(1-\widetilde{K}_{b}+\delta K_{-b}\right) /\left(q \widetilde{K}_{b}-q \delta K_{-b}+b-\varepsilon\right)+O_{1}(\varepsilon)<\widetilde{K}_{b}+ \\
\left(1-\widetilde{K}_{b}\right) w\left(\frac{1-\varepsilon}{d}\right)\left[w\left(\frac{1}{d}\right)\right]^{-1} \\
\leq \widetilde{K}_{b}+\left(1-\widetilde{K}_{b}\right)(1-\nu(\varepsilon))
\end{array}
$$

for some given smooth function $\nu$. Using 25 we arrive at the inequality

$$
\frac{b(1-\psi(\varepsilon))}{(q \psi(\varepsilon)+b-\varepsilon)}+O_{1}(\varepsilon)<1-\left(1-\widetilde{K}_{b}\right) \nu(\varepsilon)
$$

This inequality can be satisfied if only $\psi(\varepsilon) \geq C(\varepsilon+\nu(\varepsilon))$ with $C>0$ sufficiently large.

In the last step we have to prove that $\delta r(x, \tau)\left(1-\widetilde{K}_{b}\right) \leq(\widetilde{R}+\delta r(x, \tau) \exp (-\varepsilon \tau)) \delta k_{+b}+$ $a^{-1} w\left(\frac{1-\varepsilon}{d}\right) \delta k_{+b}$ for all $\delta r(x, \tau) \in\left(-\delta R_{-}, \delta R_{+}\right)$. As $\delta k_{+b}=\delta K_{+b}>0$ and $\delta R_{-}<\widetilde{R}$ then $\delta K_{+b}(\widetilde{R}+\delta r(x, \tau))>0$ for $\delta r(x, \tau) \in\left(-\delta R_{-}, \delta R_{+}\right)$. Thus we have only to prove that $\delta r(x, \tau)(1-$ $\left.\widetilde{K}_{b}\right) \leq a^{-1} w\left(\frac{1-\varepsilon}{d}\right) \delta K_{+b}$. Obviously, it suffices to show it for $\delta r=\delta r_{+}$. Let us recall that

$$
\delta r_{+}=\delta K_{+b}\left(W_{R}+\varepsilon\right)=\delta K_{+b} \frac{q(P-\widetilde{R})}{q \psi(\varepsilon)+b-\varepsilon}+\delta K_{+b} \varepsilon .
$$

Hence we have to prove that

$$
\left[\frac{q(P-\widetilde{R})}{q \psi(\varepsilon)+b-\varepsilon}+\varepsilon\right]\left(1-\widetilde{K}_{b}\right)<a^{-1} w\left(\frac{1-\varepsilon}{d}\right) .
$$


Using 28 and 33 we obtain as before that we have to satisfy the condition

$$
\frac{b}{q \psi(\varepsilon)+b-\varepsilon}+O(\varepsilon)<\frac{w\left(\frac{1-\varepsilon}{d}\right)}{w\left(\frac{1}{d}\right)} .
$$

This is implied by the inequality:

$$
\frac{b}{q \psi(\varepsilon)+b-\varepsilon}+O(\varepsilon)<1-\nu(\varepsilon)
$$

where $\nu(\varepsilon) \rightarrow 0$ as $\varepsilon \rightarrow 0$. As before, this condition can be satisfied, if only $\psi(\varepsilon) \geq C(\varepsilon+\nu(\varepsilon))$ with $C>0$ sufficiently large. Finally, taking into account what we have shown and using Theorem 2.1.2 from Pao 1992, we come to a conclusion that $-\delta r_{-}<\delta r(x, \tau)<\delta r_{+}$and $\delta k^{-}(\rho(x)) \leq \delta k(x, \tau) \leq$ $\delta k^{+}(\rho(x))$ for all $\tau \in(0, \infty)$.

So, due to the definition 18 we conclude that $\delta K(x, \tau)$ and $\delta R(x, \tau)$ tend to zero in the supremum norm as $t \rightarrow \infty$.

We have thus shown that, if the initial data $K(x, 0)$ and $R(0)$ are positive, then the solution $(K(x, \tau), R(\tau))$ to system 4-6 tends to the unique spherically symmetric solution $(\widetilde{K}, \widetilde{R})$ as $\tau \rightarrow \infty$. 


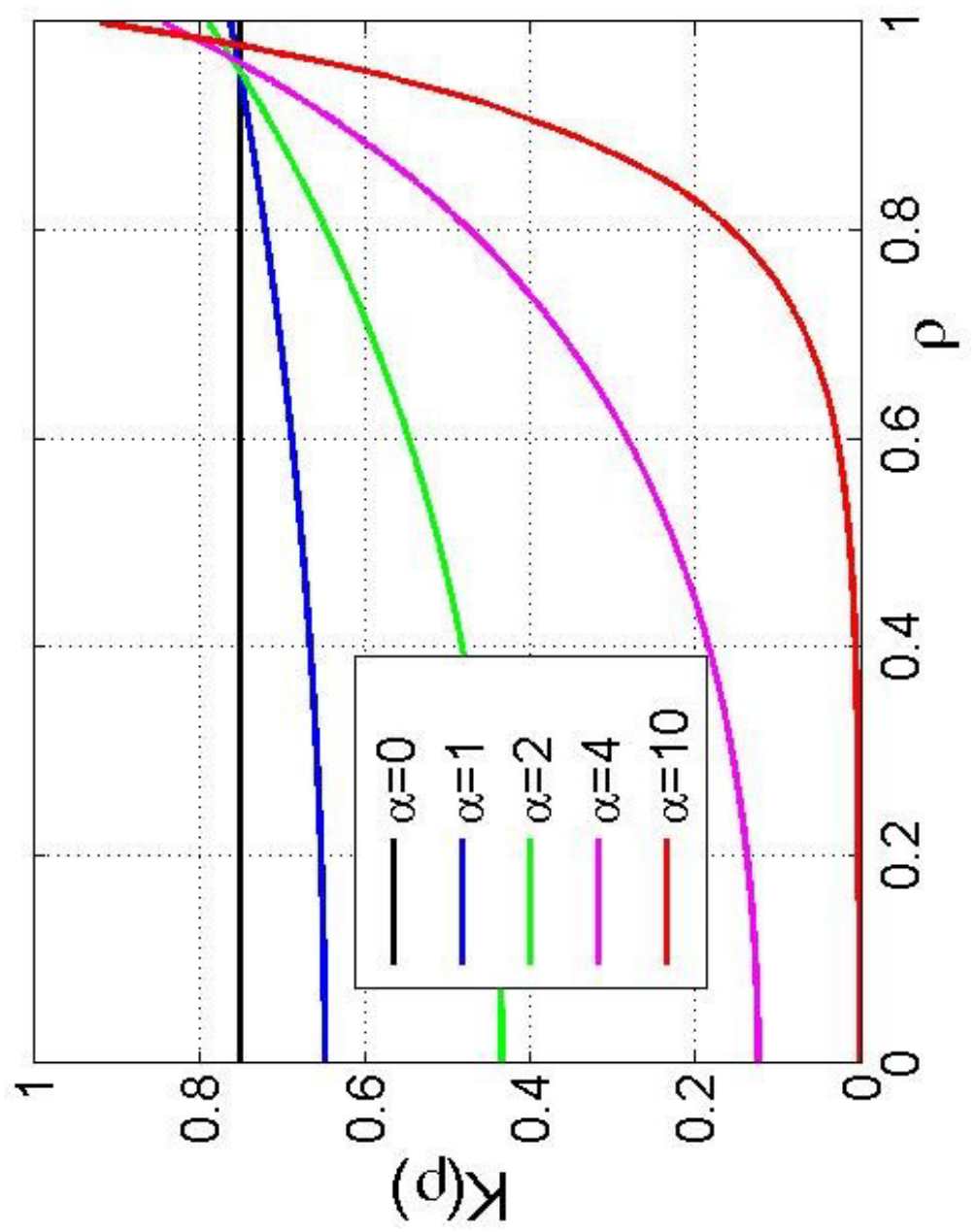

$\underset{\dot{D}}{\dot{0}}$ 

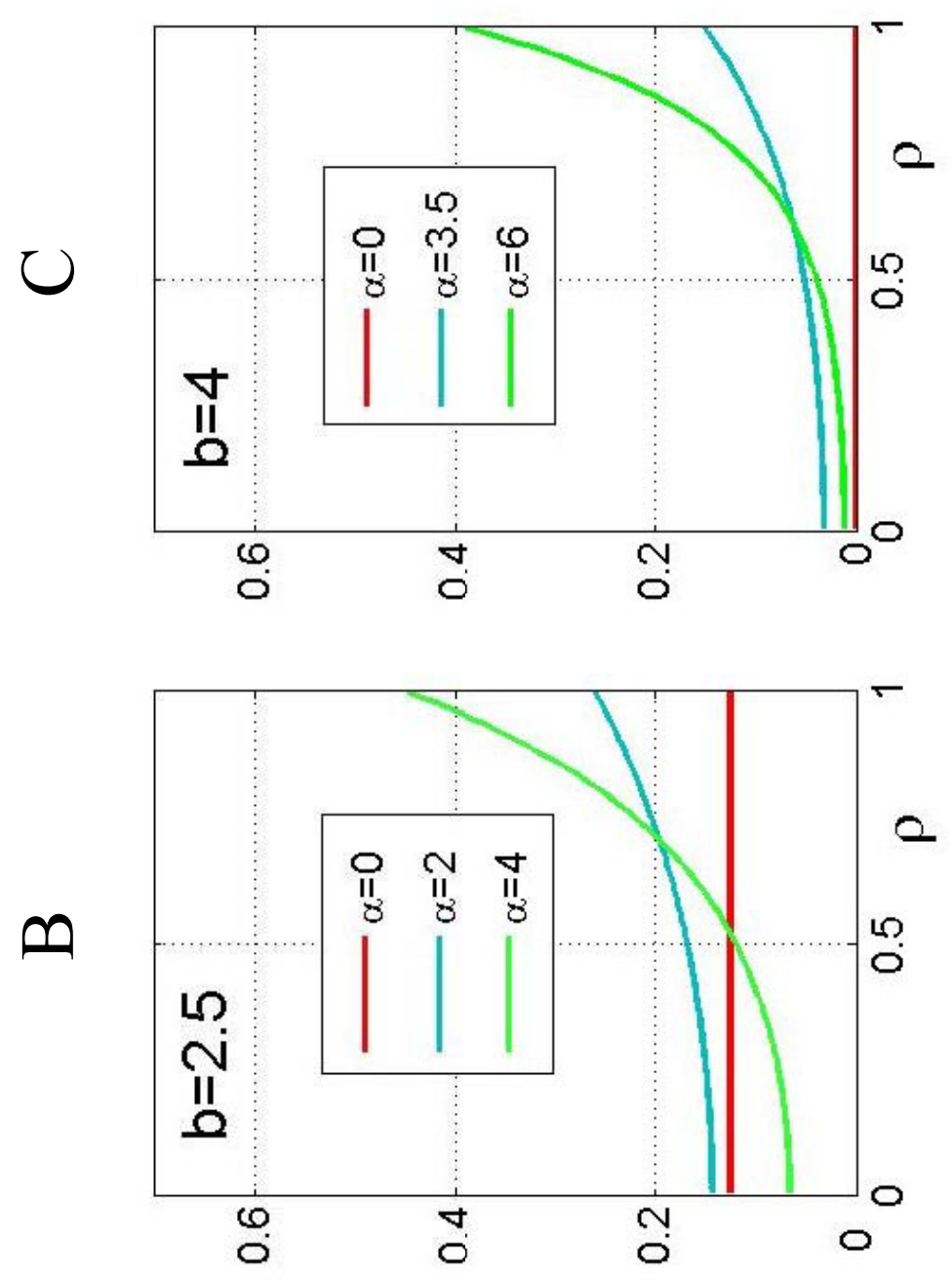

N

i

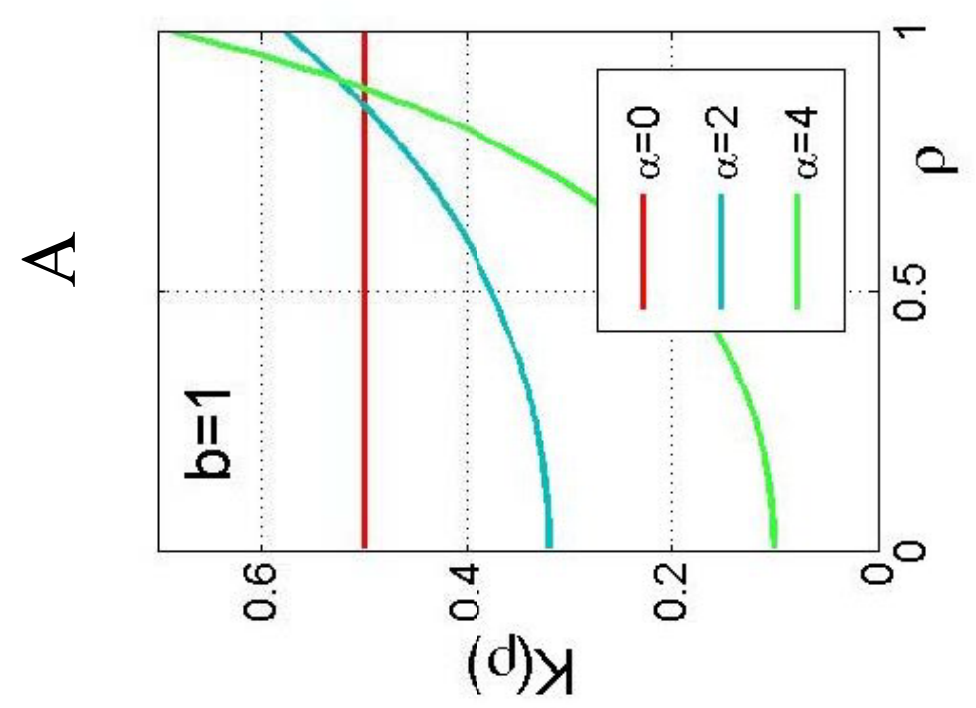



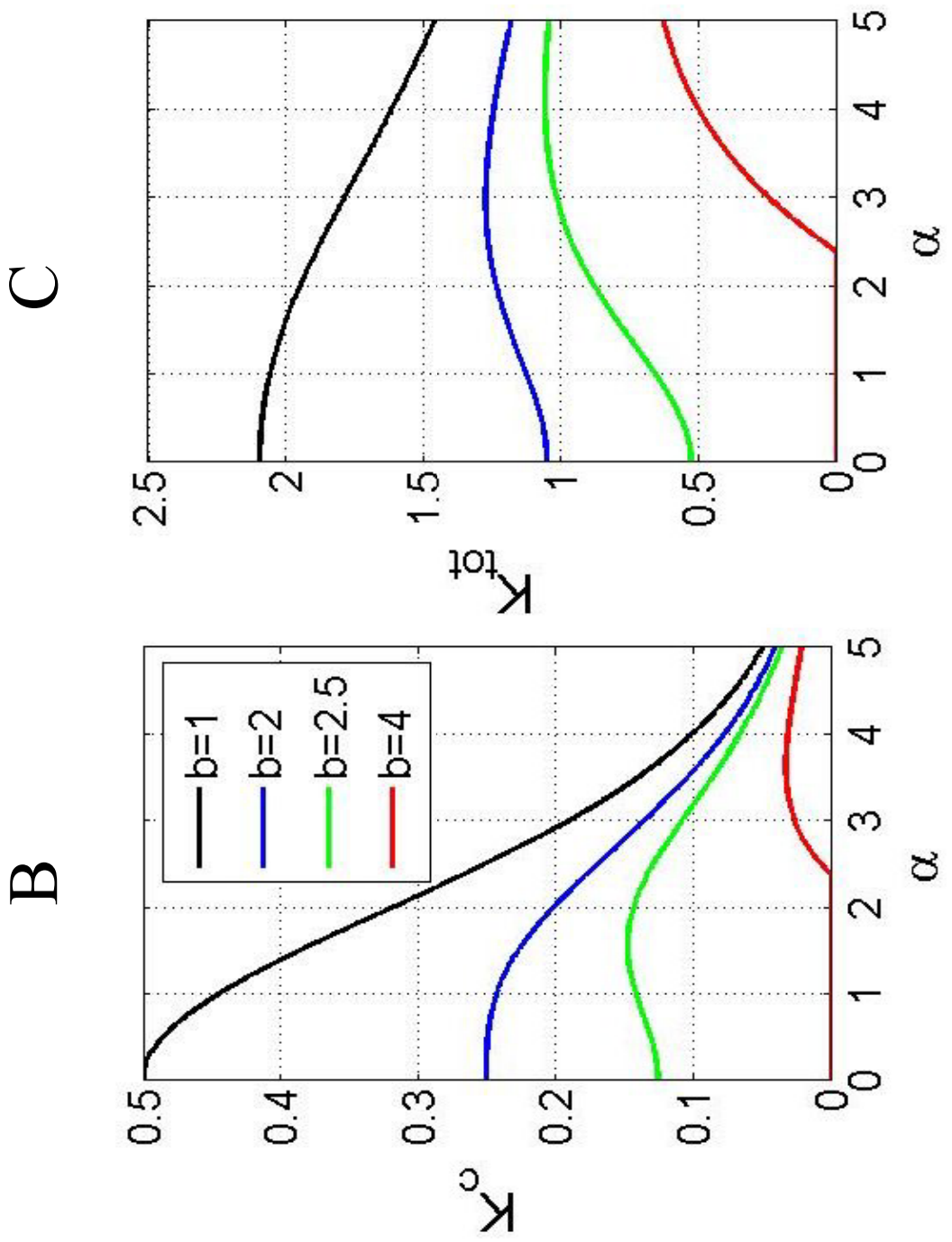

m

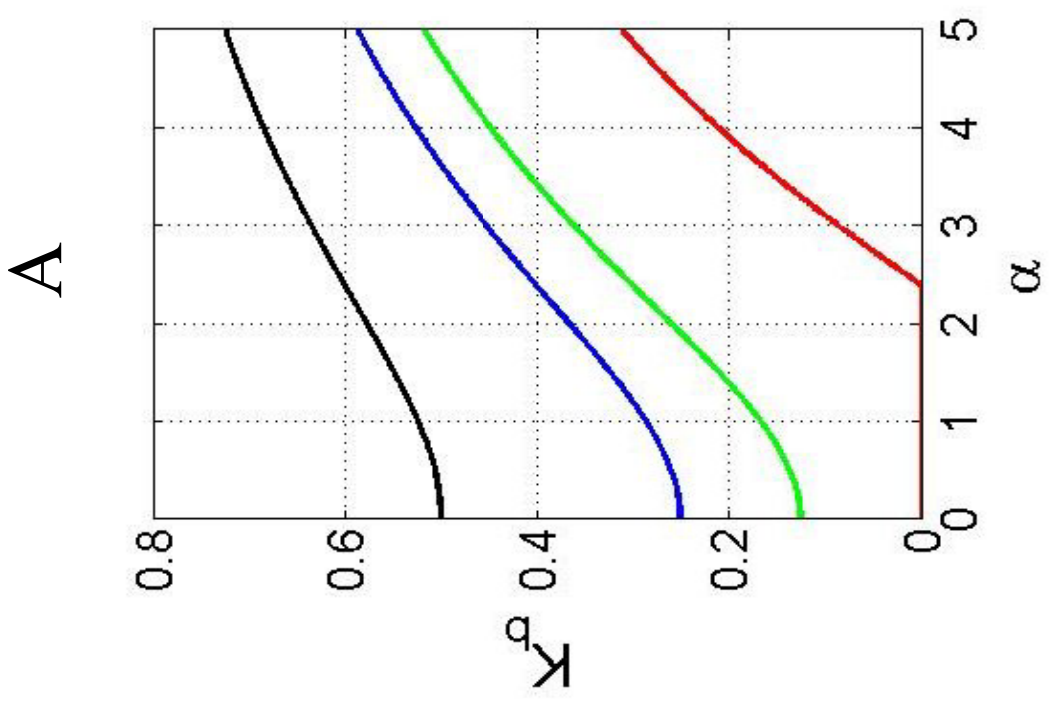




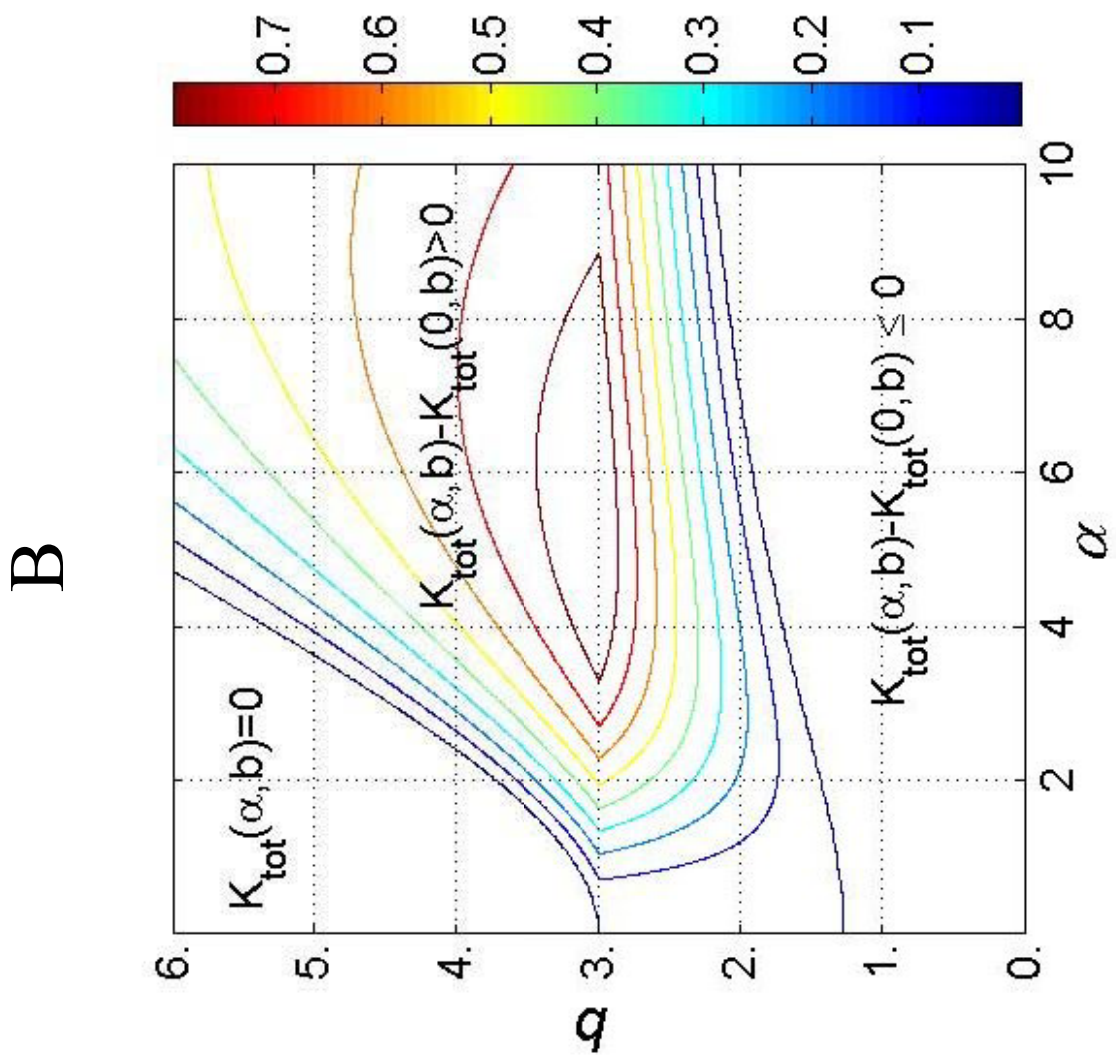

홍 웅 붕 형

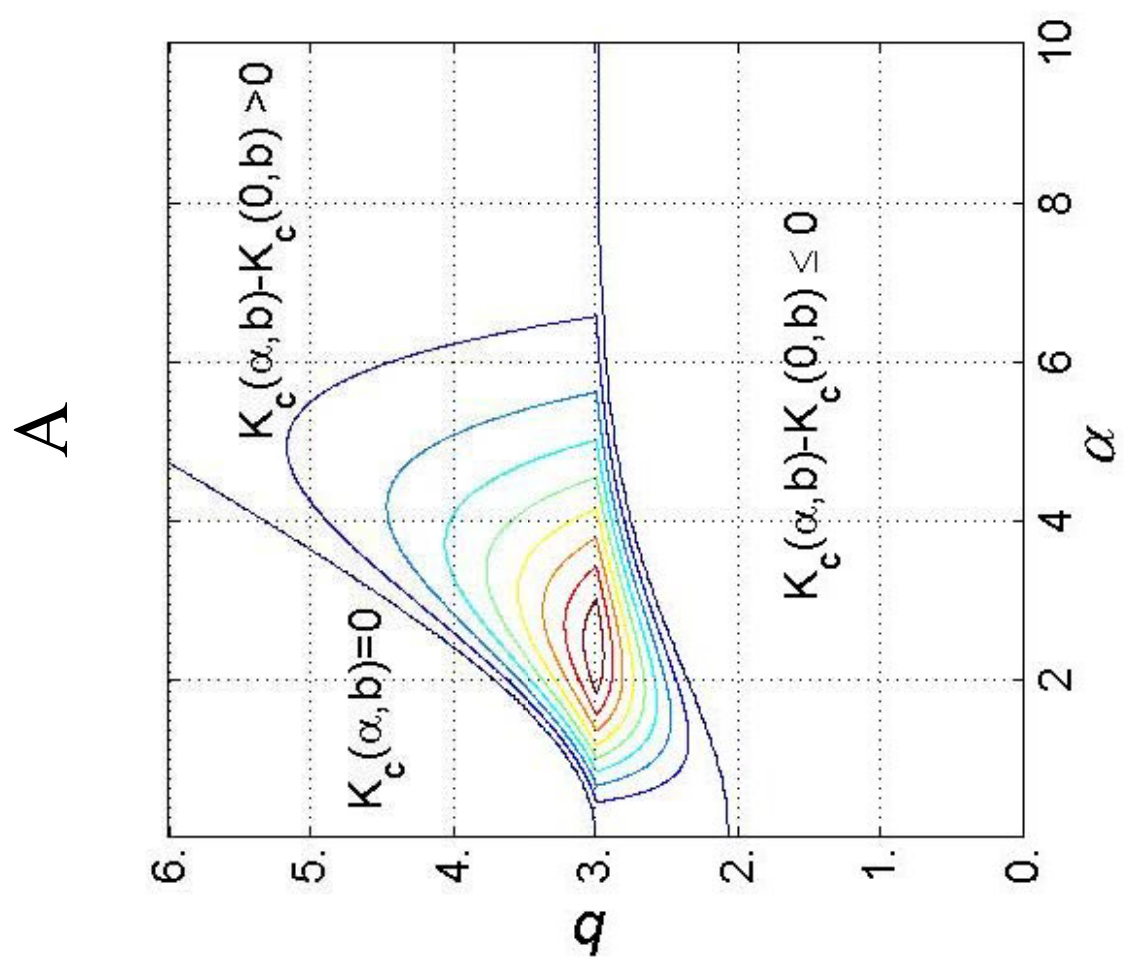

